\title{
Down-Regulation of 14-3-3б Reduces Proliferation of Human Lung Cancers But Not Colon Cancer Cells
}

\author{
Somrudee Nunun, M.Sc. ${ }^{1,2}$, Paramee Thongsuksai, M.D. ${ }^{1-3}$, Keson Trakunrum, M.Sc. ${ }^{1,2}$, \\ Pritsana Raungrut, Ph.D.,
}

${ }^{1}$ Department of Biomedical Sciences, ${ }^{2}$ The Excellent Research Laboratory of Cancer Molecular Biology, ${ }^{3}$ Department of Pathology, Faculty of Medicine, Prince of Songkla University, Hat Yai, Songkhla 90110, Thailand.

Received 18 May 2017 • Accepted 30 August 2017 • Published online 24 May 2018

\section{Abstract:}

Objective: $14-3-3 \sigma$ protein is well known for its tumor suppressive function in breast cancer. However, recent evidence has raised the possibility that the 14-3-30 protein may also have an oncogenic function in certain cancer types. The aim of this study was to investigate the oncogenic function of 14-3-30 in adenocarcinoma cell lines of the lung (A549, H358) and colon (HT-29).

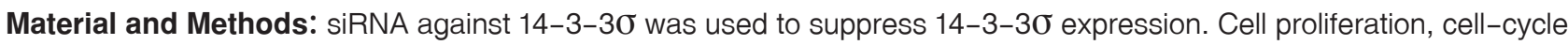
distribution and expression of related molecules were determined by MTT, flow cytometry, and western blotting, respectively. Results: Down-regulation of $14-3-3 \sigma$ significantly reduced the proliferation of A549 and $\mathrm{H} 358$ by $35.0 \%$ and $31.0 \%$, respectively, and significantly induced cell cycle arrest at the G0/G1 and G2 M phases, respectively. Increased p21

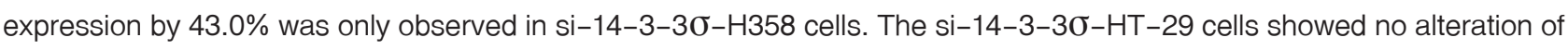
cell proliferation and cell-cycle distribution but harbored reduced p53 expression by $56.0 \%$ and p27 expression by $67.0 \%$. Conclusion: 14-3-30 may have an oncogenic function in lung adenocarcinoma but play a different role in colon cancer.

Keywords: $14-3-3 \sigma$, cancer, cell proliferation, siRNA 


\section{Introduction}

14-3-3 proteins are a family of conserved regulatory proteins that are expressed in all eukaryotic cells. ${ }^{1}$ The 14-3-3 proteins contain a phosphorylated serine, or threonine residues that preferentially bind to various binding proteins. This, binding of 14-3-3 proteins has diverse effects which depends on the binding partners. ${ }^{2,3}$ In mammalian cells, the 14-3-3 proteins can be classified into 7 isoforms, identified as $\beta, \gamma, \varepsilon, \eta, \sigma, \tau$, and $\zeta^{1,4}$ Among these isoforms, $14-3-3 \sigma$ has been shown to be involved in cellular processes such as the deoxyribonucleic acid (DNA) damage response, cell proliferation, cell cycle, and apoptosis. ${ }^{2,5}$ Although, much recent evidence has indicated an as sociation between 14-3-3 $\sigma$ and cancer development, the exact role of this protein are as yet unknown.

Functionally, $14-3-3 \sigma$ has been reported to act as both as a tumor suppressor and an oncogene in various cancers. Loss of expression of 14-3-30 expression has been observed in breast cancer ${ }^{6}$ and ovarian, prostate, and endometrial cancer, ${ }^{7}$ while being found in increased levels in colon cancer, ${ }^{8}$ lung cancer ${ }^{9}$ and pancreatic cancer. ${ }^{10}$ A previous studies has demonstrated that 14-3-30 functions as a tumor suppressor by exerting positive regulation on p53 activity resulting in inhibition of tumor growth. ${ }^{11}$ Elevated expression of $14-3-3 \sigma$ has been shown to arrest the cell cycle at the G2 phase of colon and breast cancer cells. ${ }^{12,13}$ By contrast, in an oncogenic role, ectopic expression of $14-3-3 \sigma$ has been reported to promote proliferation of lung cancer cells, ${ }^{14}$ and motility or invasion of colon ${ }^{15}$ and breast ${ }^{16}$ cancer cells. These different roles have also been demonstrated in a clinical setting. Decreased expression of 14-3-30 has been associated with a poor prognosis in cholangiocarcinoma, ${ }^{17}$ breast carcinoma $^{18}$ and uterine serous carcinoma ${ }^{19}$ whereas $^{2}$ increased expression of 14-3-3 $\sigma$ in colon, ${ }^{8}$ pancreatic, ${ }^{10}$ and gastric ${ }^{20}$ cancers has been associated with a poor prognosis.
Overall, these evidences indicate that $14-3-3 \sigma$ plays different roles in tumorigenesis in different types of cancers. In this present study, specifically targeted siRNA was used to down-regulate the expression of $14-3-3 \sigma$ in lung (A549 and H358) and colon (HT-29) cancer cells to investigate the effect of $14-3-3 \sigma$ on cell proliferation.

\section{Material and Methods}

\section{Cell culture}

The A549 and H358 human lung cancer cell lines were purchased from the American Type Culture Collection (Manassas, VA, USA) and cultured in RPMI 1640 medium (Gibco, Grand Island, USA). The HT-29 human colon cancer cell line was a generous gift from Dr.Potchanapond Graidist and maintained in DMEM medium (Gibco, Grand Island, USA). All media were supplemented with $10.0 \%$ fetal bovine serum (FBS), $100 \mathrm{U} / \mathrm{ml}$ penicillin, and $100 \mu \mathrm{g}$ / $\mathrm{ml}$ streptomycin (Gibco, Grand Island, USA). Cells were incubated at $37^{\circ} \mathrm{C}$ in a $5 \% \mathrm{CO}_{2}$ humidified atmosphere.

\section{Silencing of $14-3-3 \sigma$ expression}

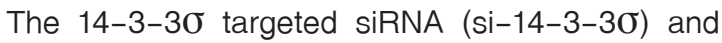
non-targeting siRNA (si-control) (Thermo Fisher Scientific, MA, USA) were diluted separately in Opti-MEM I (Gibco, Grand Island, USA) to final concentration of $40 \mathrm{nM}$. Transient transfection of siRNA was performed using Lipofectamine transfection reagent 2000 (Invitrogen, CA, USA) according to the manufacturer's protocol. The A549 ( $2 \times 10^{5}$ cells/well) and H358 ( $3 \times 10^{5}$ cells/well) cells were suspended in antibiotic-free medium. Then the cell suspensions were combined with si-14-3-30 or si-control containing transfection reagent and seeded in a 6 -well plate. For the HT-29, the cells $\left(1 \times 10^{5}\right.$ cells/ well) were seeded in a 24-well plate and cultured for $24 \mathrm{hr}$, until the cells reached $50.0-60.0 \%$ confluence. Pre-plated cells were incubated with a transfection mixture containing si-14-3-30 or si-control. 
The media were replaced at $6 \mathrm{hr}$ for A549 and H358 and at $24 \mathrm{hr}$ for HT-29. The cells were grown in a $5 \% \mathrm{CO}_{2}$ humidified atmosphere at $37{ }^{\circ} \mathrm{C}$ and harvested at $48 \mathrm{hr}$ (A549 cells) or $72 \mathrm{hr}$, (H358 and HT-29 cells) after transfection. Cells without treatment (Cells) and transfected cells with only transfection reagent (Mock) were assigned as controls.

\section{Cell proliferation assay}

The 3-(4, 5-dimethyl thiazol-2-yl)-2, 5-diphenyl tetrazolium bromide (MTT) assay was used to determine the proliferation of cells. After siRNA transfection as mentioned above, the cells were trypsinized and seeded in a 96-well plate for $24 \mathrm{hr}$ at density of $2 \times 10^{4}, 3 \times 10^{4}$, or $1 \times 10^{4}$ cells/well for the A549, H358 or HT-29 cells, respectively. The cells were washed and treated with $100 \mu \mathrm{l}$ of media containing $0.5 \mathrm{mg} / \mathrm{ml}$ MTT solution (Invitrogen, CA, USA) for $2 \mathrm{hrs}$ at $37^{\circ} \mathrm{C}$. The media were removed and reaction was stopped by the addition of $100 \mu \mathrm{L}$ DMSO (Amresco, OH, USA). The reaction products were quantified by measuring the absorbance at 550 and $650 \mathrm{~nm}$ using a microplate reader (Molecular Devices, CA, USA). The optical density value of the cells without treatment was considered as $100.0 \%$ cell proliferation. Cell growth in the other groups was expressed as a percentage by comparing them with cells without treatment.

\section{Cell cycle analysis}

After siRNA transfection, cells were trypsinized and washed with ice-cold phosphate-buffered saline (PBS). The cells $\left(2.5-5 \times 10^{5}\right.$ cells) were fixed in ice-cold $70.0 \%$ ethanol and incubated at $-20{ }^{\circ} \mathrm{C}$ for at least $3 \mathrm{hr}$. The fixed cells were centrifuged at $300 \mathrm{~g}$ for $5 \mathrm{~min}$ and washed with $200 \mu \mathrm{l}$ PBS. The cells were then resuspended in $200 \mu \mathrm{l}$ of Muse ${ }^{\mathrm{TM}}$ cell cycle reagent containing propidium iodide at room temperature in the dark for $30 \mathrm{~min}$ and analyzed using the Muse ${ }^{\circledR}$ Cell Analyzer (Merck Millipore, CA, USA).

\section{Western blotting}

Cells were lysed with a radioimmunoprecipitation assay (RIPA) buffer (EMD Millipore, CA, USA) containing freshly prepared a protease inhibitor cocktail (Roche, Beijing, China). Protein concentrations were determined using the Bradford assay (Bio-Rad Laboratories, Hercules, USA). Total protein $(30 \mu \mathrm{g})$ was loaded into a $12.0 \%$ sodium dodecyl sulfate polyacrylamide gel electrophoresis (SDSPAGE) gel solution and transferred to a nitrocellulose membrane (Bio-Rad Labora-tories, Hercules, USA) which was then blocked with $3.0 \%$ BSA for $1 \mathrm{hr}$ and probed with 1:500 of mouse anti-14-3-30 (Santa Cruz Biotechnology, CA, USA), mouse anti-p21NAF1/Cip1 (MD Millipore, Billerica, USA), and rabbit anti-p27 (MD Millipore, Billerica, USA), rabbit anti-p53 antibodies and 1:1000 of rabbit anti- $\beta$-actin (Cell Signal-ing Technology, MA, USA) antibody overnight at $4^{\circ} \mathrm{C}$.

After washing, the membranes were incubated with either horseradish peroxidase conjugated goat anti-mouse or rabbit antibodies (1:2,000; MD Millipore, Billerica, USA) for 1-2 hr at room temperature. Protein expressions were detected with chemiluminescence SuperSignal West Pico or Dura chemiluminescent substrate (Thermo Fisher Scientific, MA, USA). Protein bands were visualized using a couple-charged device (CCD) camera (Vilber Lourmat, Eberhardzell, Germany). Intensities of the bands were quantified using CCD software (Biogenomed, CA, USA). The relative expression of each protein was deter mined by normalization with internal control $\beta$-actin.

\section{Statistical analysis}

Three independent experiments were carried out. Data were expressed as mean \pm standard deviation (S.D.). 
The differences of outcomes between the si-14-3-30 group and the control groups, (cells, mock, and si-control) were analyzed by Student's independent t-test. A p-value less than 0.05 was considered statistically significant.

\section{Results}

Effect of down-regulation of $14-3-3 \sigma$ on cell proliferation

14-3-30 levels following siRNA silencing were analyzed by western blotting. Such analysis revealed that the 14-3-30-targeted siRNA effectively silenced 14-3-30 expression in A549, H358, and HT-29 cells by $59.0 \%, 51.0 \%$, and $44.0 \%$, respectively (Figure 1A). In A549, decreased 14-3-30 expression resulted in inhibition of cell proliferation by $35.0 \%, 36.0 \%$, and $30.0 \%$ compared with non-transfected cells $(p-$ value $=0.013)$, mock $(p-$ value $=$ 0.018 ) and si-control ( $p-v a l u e=0.040)$, respectively. Similar to $\mathrm{A} 549$, the si-14-3-30-H358 cells revealed a significant reduction in cell proliferation by $31.0 \%, 20.0 \%$, and $44.0 \%$ compared with the non-transfected cells ( $p$-value $=0.013$ ), mock ( $p$-value=0.021) and si-control ( $p$-value=0.013), respectively. By contrast, down- regulation of the 14-3-30 did not have any effect on cell proliferation in the HT29 cells (Figure 1B).

\section{Effect of down-regulation of $14-3-3 \sigma$ on cell} cycle

To elucidate the effect of 14-3-30 down-regulation in mediating cell cycle arrest, cell cycle analysis after 14-3-30 knockdown was performed by flow cytometry (Figure 2). In A549, the results showed that G0/G1-phase DNA content was significantly increased in si-14-3-30 transfected cells by approximately $7.0 \%$ compared with the non-transfected cells ( $p$-value $=0.015)$ and significant reductions in $\mathrm{S}$-phase by $3.0 \%(p-$ value $=0.032)$ and in G2M-phase by $3.2 \%$ ( $p$-value=0.017) compared with the non-transfected cells were also found.
(A)
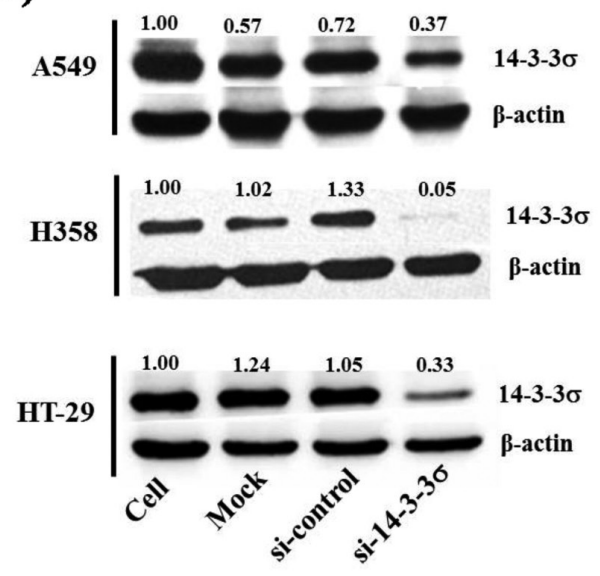

(B)

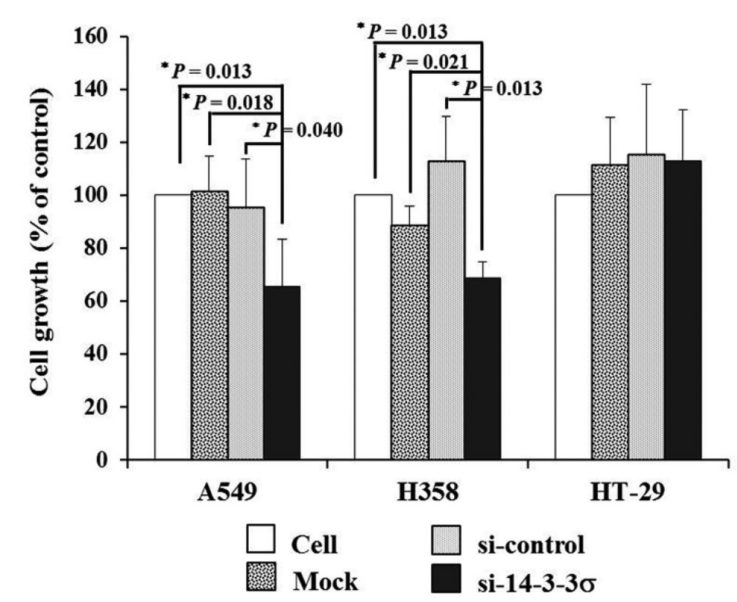

${ }^{*} p$-value $<0.05$ is considered as a significant difference from the controls.

Figure 1 Effects of siRNA against 14-3-30 in the expression of $14-3-3 \sigma$ protein by western blot analysis (A) and MTT proliferation assay (B) in A549, H358 and HT-29 cells (si-14-3-30) compared to untransfected cells (Cell), transfected cells with only transfection reagent (Mock) and transfected cells with non-targeting siRNA (si-control). Untransfected cells are considered as $100.0 \%$ cell proliferation. 
(A)

(B)
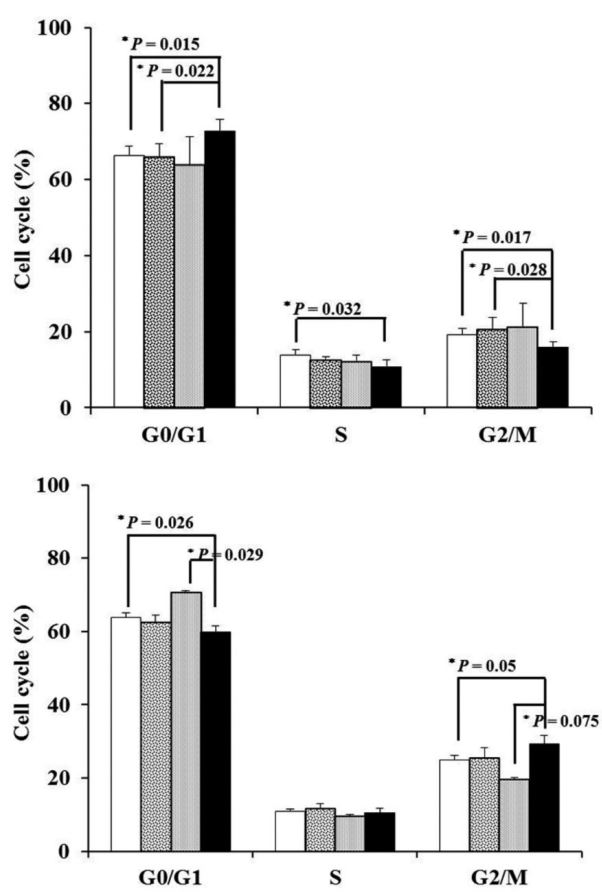

(C)

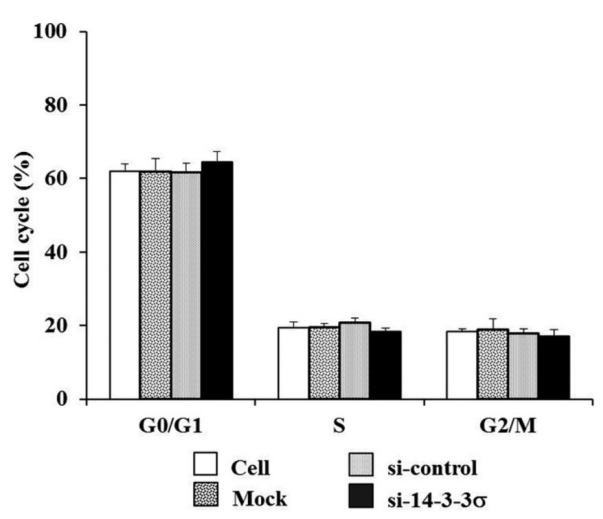

* $p$-value $<0.05$ is considered as a significant different from the controls.

Figure 2 Effect of siRNA against 14-3-3 $\sigma$ on cell cycle by flow cytometry in A549 (A), H358 (B) and HT29 (C) compared to untransfected cells (Cell), transfected cells with only transfection reagent (Mock) and transfected cells with non-targeting siRNA (si-control). Data are expressed as mean \pm S.D. from three independent experiments.
By contrast, the si-14-3-30-transfected H358 cells showed a $4.0 \%$ decrease in the G0/G1-phase DNA content compared with that of the non-transfected cells $(p$-value $=0.026)$ and an increase in DNA content of the G2/M-phase by $4.0 \%$ when compared with the nontransfected cells ( $p$-value $=0.050$ ). However, siRNA-transfected HT-29 cells did not show significant alteration of DNA content compared with the controls.

Effect of down-regulation of $14-3-3 \sigma$ on cell cycle regulatory proteins

To examine the effect of 14-3-30 down-regulation on cell cycle regulatory proteins, expressions of p21, p27, and p53 were evaluated by western blotting. In lung cancer cells, there were no significant differences of p21 and p27 expressions in si-14-3-3 $\sigma$ transfected A549 (Figure 3A) whereas there was a significant increase of p21 expression in si-14-3-30 transfected $\mathrm{H} 358$ by $43.0 \%$ compared to the non-transfected cells ( $p-$ value=0.015) (Figure 3B).

For HT-29 colon cancer cells, dramatic reductions of p53 and p27 expression in transfected cells compared to the controls were observed. The si-14-3-30-HT-29 cells showed decreased p53 expression by $56.0 \%$ compared to the non-transfected cells ( $p$-value=0.010). The p27 expression was also markedly reduced by $67.0 \%$ in the transfected cells when compared with the nontransfected cells. The p53 protein was not detected in A549 and H358. Similarly, the p27 and p21 proteins were not detected in H358 and HT29, respectively (Figure 3C).

\section{Discussion}

Accumulating data indicated that $14-3-3 \sigma$ participates in tumorigenesis and correlates with the prognosis. However, results from different types of cancers are different. In this study, we evaluated the role of $14-3-3 \sigma$ on cell 
(A)
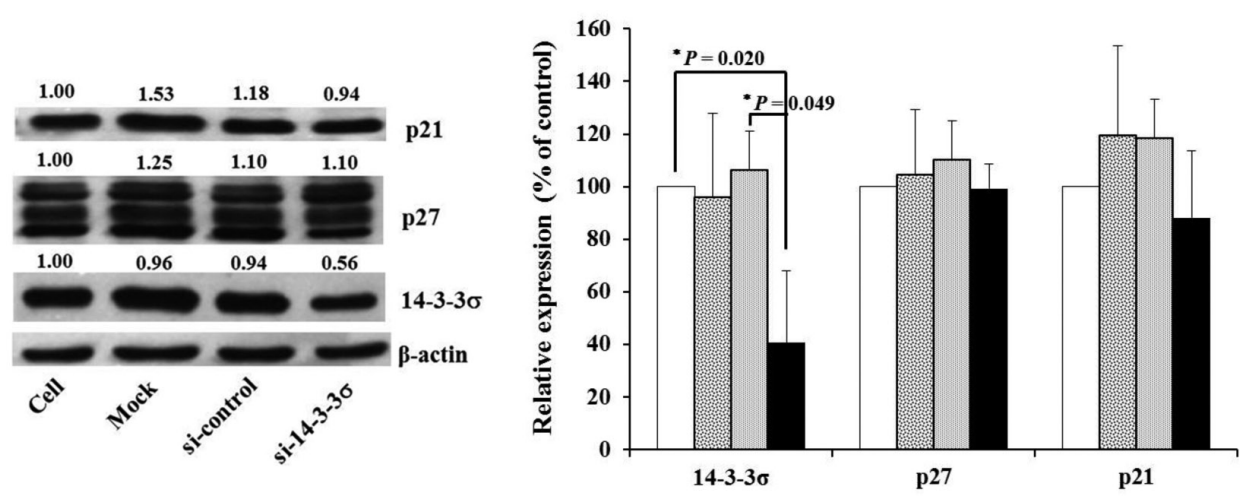

(B)
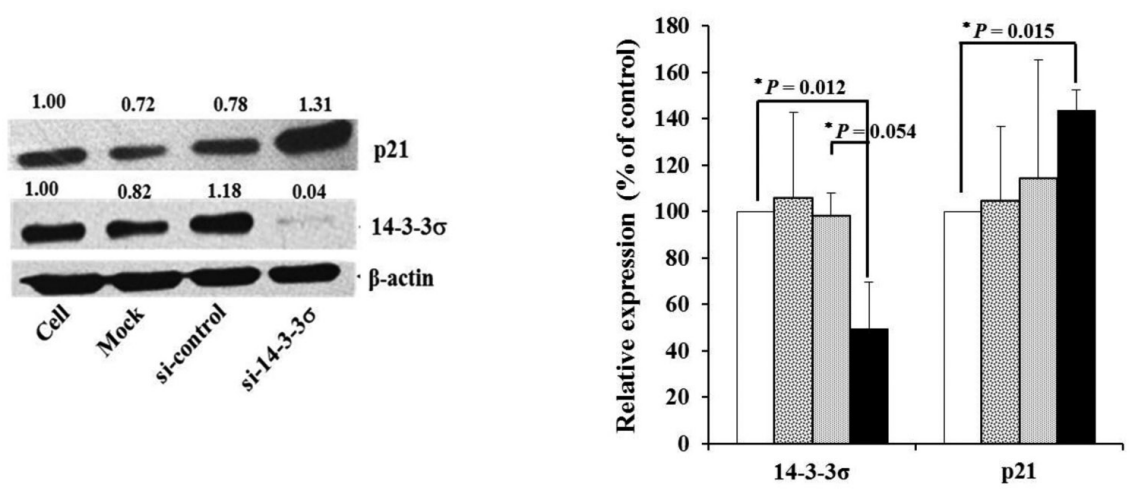

(C)
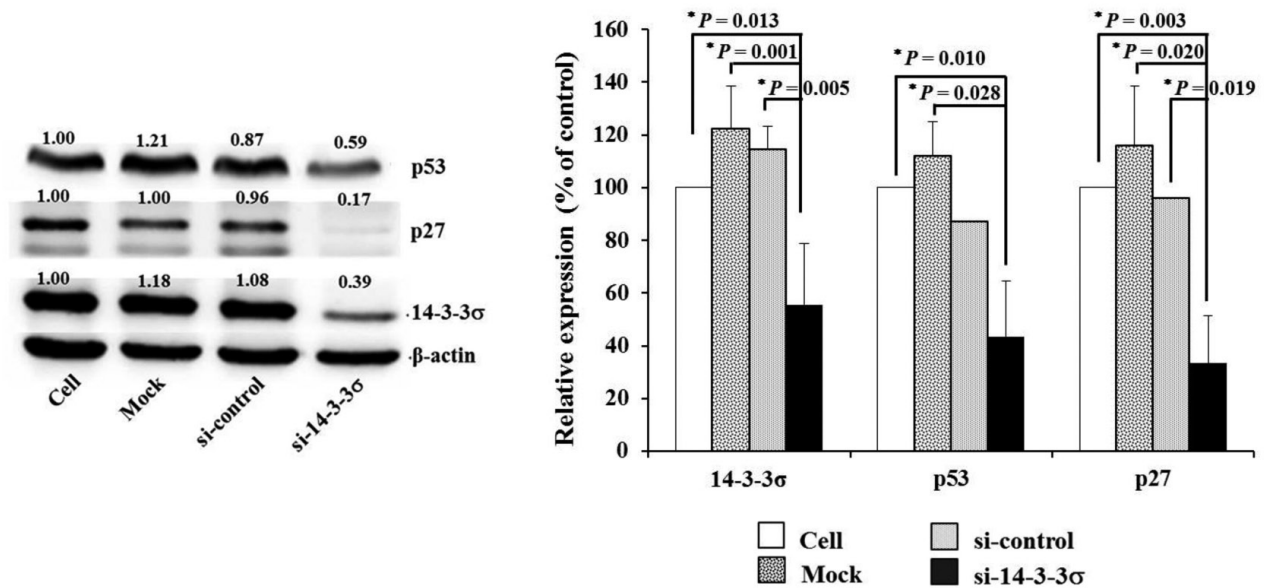

${ }^{*} \mathrm{p}$-value $<0.05$ is considered as a significant different from the controls.

Figure 3 Effect of siRNA against 14-3-3 0 in the expressions of p21, p27 and p53 by western blotting in A549 (A), H358 (B) and HT-29 (C) cells compared to untransfected cells (Cell), transfected cells with only transfection reagent (Mock) and transfected cells with non-targeting siRNA (si-control). Data are expressed as mean \pm S.D. from three independent experiments. 
proliferation in lung and colon cancer cell lines. Our results revealed that down-regulation of $14-3-3 \sigma$ significantly reduced proliferation of lung cancer cells and induced cell-cycle arrest at the G0/G1 and G2/M phases in $\mathrm{A} 549$ and $\mathrm{H} 358$, respectively, while there was no effect on cell proliferation of colon cancer cells.

$14-3-3 \sigma$ has been proposed as an oncogene in lung cancer. Increased expression of the 14-3-30 has been observed in lung cancer ${ }^{21}$ and it has also been associated with progression from in situ to invasive adenocarcinoma of the lung. ${ }^{22}$ In addition, Shiba-Ishii et $\mathrm{al}^{22}$ found that the expression of $14-3-3 \sigma$ induced proliferation of A549 cells whereas down-regulation of this protein significantly decreased their proliferation. Our results are consistent with this report, indicating that $14-3-3 \sigma$ may function as an oncogene in lung adenocarcinoma. In colon cancer, oncogenic and tumor suppressor functions have been reported. Ide et al. ${ }^{15}$ found that migration was activated in colorectal carcinoma cells with high expressions of $14-3-3 \sigma$. In contrast, other studies have shown that $14-3-3 \sigma$ induces G2 arrest and inhibits proliferation of colorectal cancer cells. ${ }^{12,23,24}$ Additionally, some studies have found that 14-3-30 negatively regulated the cell cycle through cyclindependent kinase association resulting in preventing these proteins from entering the nucleus. ${ }^{24}$ However, this present study found no effect on cell proliferation in colon cancer cells after the silencing of $14-3-3 \sigma$.

Previous studies have also shown that $14-3-30$ and p53 are functionally linked. Thus, a reduction of cancer cells after the silencing of 14-3-30 may be associated with p53 function. Yang et al. ${ }^{11}$ have demonstrated that 14-3-30 positively regulated p53 resulting in the arrest of cells, we expected that phase of the cell cycle would be effected after the silencing of $14-3-3 \sigma$ in human cancer cell lines with differing p53 statuses, including A549 (p53 wild-type), H358 (p53 deleted), and HT-29 (p53 mutated). However, we could not detect the endogenous wide-type p53 expression in A549. It may be that p53 is naturally unstable and prone to degradation through the Mdm2-mediated p53 ubiquitination. ${ }^{25}$ In contrast, the ubiquitinating process of p53 is impaired in mutanttype p53 cells26 and this result in accumulation of mutated p53 protein in HT-29 cells.

Interestingly, although the down-regulation of $14-3-3 \sigma$ had a significant effect to the reduction of the proliferation of lung cancer cells, there were no apparent changes in p21 and p27 expressions which are downstream molecules of p53 in A549. It may indicate that the proliferation of lung cancer cells is not due to the p53-associated 14-3-3 $\sigma$ expression and it is possible that $14-3-3 \sigma$ regulates cell proliferation by other mechanisms. Chang et al. $^{27}$ have demonstrated that

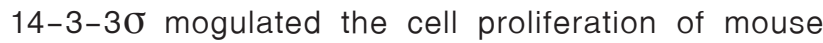
embryonic stem cells through regulation of GSK-3 $\beta$ and $\beta$-catenin. Furthermore, an increased expression of p21 in H358 cells harboring a p53 deletion after silencing of 14-3-30 implies p53-independent regulation of p21 expression. We speculated that $14-3-3 \sigma$ can directly bind to p21 and a decrease of 14-3-30 may diminish binding of $14-3-3 \sigma$ to p21 contributing to an increase of p21 and promoting cell cycle arrest in H358 cells.

In colon cancer, although we did not observed any alteration in cell proliferation, a reduction of p53 and p27 expressions was shown. Casalini et al. ${ }^{28}$ have shown that the p53 regulated the expression of p27. Thus, it is possibly that the decreased expression of p53 after silencing of $14-3-3 \sigma$ affects the p27 expression. This alteration is not related to the cell proliferation of colon cancer, but may be involved with other functions. For example, Besson et al. have shown that p27 regulated cell migration of mouse embryonic fibroblasts via modulation of the Rho pathway. ${ }^{29}$ 


\section{Conclusion}

In conclusion, our results support the hypothesis that 14-3-30 may serve as an oncogene in lung adenocarcinoma, and it may play a different role in colon cancer.

\section{Acknowledgement}

This study was funded by a grant from Prince of Songkla University and the Faculty of Medicine, Songkhla, Thailand. The Excellent Research Laboratory of Cancer Molecular Biology provided research facilities.

\section{References}

1. van Heusden GP. 14-3-3 proteins: regulators of numerous eukaryotic proteins. IUBMB Life 2005;57:623-9.

2. van Hemert MJ, Steensma HY, van Heusden GP. 14-3-3 proteins: key regulators of cell division, signalling and apoptosis. Bioessays 2001;23:936-46.

3. Fu H, Subramanian RR, Masters SC. 14-3-3 proteins: structure, function, and regulation. Annu Rev Pharmacol Toxicol 2000; 40:617-47.

4. Aitken A. 14-3-3 proteins: a historic overview. Semin Cancer Biol 2006;16:162-72.

5. Lodygin D, Hermeking $\mathrm{H}$. The role of epigenetic inactiva-tion of 14-3-3 sigma in human cancer. Cell Res 2005;15:23746.

6. Ferguson AT, Evron E, Umbricht CB, Pandita TK, Chan TA, Hermeking $\mathrm{H}$, et al. High frequency of hypermethylation at the 14-3-3 sigma locus leads to gene silencing in breast cancer. Proc Natl Acad Sci U S A 2000;97:6049-54.

7. Mhawech P, Benz A, Cerato C, Greloz V, Assaly M, Desmond JC, et al. Downregulation of 14-3-3 sigma in ovary, prostate and endometrial carcinomas is associated with $\mathrm{CpG}$ island methylation. Mod Pathol 2005;18:340-8.

8. Perathoner A, Pirkebner D, Brandacher G, Spizzo G, Stadlmann $\mathrm{S}$, Obrist $\mathrm{P}$, et al. 14-3-3 sigma expression is an independent prognostic parameter for poor survival in colorectal carcinoma patients. Clin Cancer Res 2005;11:3274-9.

9. Osada H, Tatematsu Y, Yatabe Y, Nakagawa T, Konishi H, Harano $T$, et al. Frequent and histological type-specific inactivation of 14-3-3 sigma in human lung cancers. Oncogene 2002;21:2418-24.
10. Li Z, Dong Z, Myer D, Yip-Schneider M, Liu J, Cui P, et al. Role of 14-3-3 sigma in poor prognosis and in radiation and drug resistance of human pancreatic cancers. BMC Cancer 2010;10:598.

11. Yang HY, Wen YY, Chen CH, Lozano G, Lee MH. 14-3-3 sigma positively regulates p53 and suppresses tumor growth. Mol Cell Biol 2003;23:7096-107.

12. Hermeking $\mathrm{H}$, Lengauer $\mathrm{C}$, Polyak K, He TC, Zhang L, Thiagalingam $S$, et al. 14-3-3 sigma is a p53-regulated inhibitor of G2/M progression. Mol Cell 1997;1:3-11.

13. Laronga C, Yang HY, Neal C, Lee MH. Association of the cyclin-dependent kinases and 14-3-3 sigma negatively regulates cell cycle progression. J Biol Chem 2000;275: 23106-12

14. Shiba-Ishii A, Kim Y, Shiozawa T, Iyama S, Satomi K, Kano J, et al. Stratifin accelerates progression of lung adenocarcinoma at an early stage. Mol Cancer 2015;14:142.

15. Ide M, Saito K, Tsutsumi S, Tsuboi K, Yamaguchi S, Asao $\mathrm{T}$, et al. Over-expression of 14-3-3 sigma in budding colorectal cancer cells modulates cell migration in the presence of tenascin-C. Oncol Rep 2007;18:1451-6.

16. Boudreau A, Tanner K, Wang D, Geyer FC, Reis-Filho JS, Bissell MJ. 14-3-3 sigma stabilizes a complex of soluble actin and intermediate filament to enable breast tumor invasion. Proc Natl Acad Sci U S A 2013; 110: E3937-44.

17. Kuroda $Y$, Aishima S, Taketomi A, Nishihara Y, Iguchi T, Taguchi $K$, et al. 14-3-3 sigma negatively regulates the cell cycle, and its down-regulation is associated with poor outcome in intrahepatic cholangiocarcinoma. Hum Pathol 2007;38: 1014-22.

18. Yoon NK, Seligson DB, Chia D, Elshimali Y, Sulur G, Li A, et al. Higher expression levels of 14-3-3 sigma in ductal carcinoma in situ of the breast predict poorer outcome. Cancer Biomark 2009;5:215-24.

19. Suzuki F, Nagase S, Suzuki K, Oba E, Hiroki E, Matsuda Y, et al. Decreased expression of $14-3-3$ sigma is predictive of poor prognosis for patients with human uterine papillary serous carcinoma. Tohoku J Exp Med 2013;231:193-9.

20. Li YL, Liu L, Xiao Y, Zeng T, Zeng C. 14-3-3 sigma is an independent prognostic biomarker for gastric cancer and is associated with apoptosis and proliferation in gastric cancer. Oncol Lett 2015;9:290-4. 
21. Qi W, Liu X, Qiao D, Martinez JD. Isoform-specific expression of 14-3-3 proteins in human lung cancer tissues. Int $J$ Cancer 2005;113:359-63.

22. Shiba-Ishii A, Kano J, Morishita $Y$, Sato $Y$, Minami $Y$, Noguch M. High expression of stratifin is a universal abnormality during the course of malignant progression of early-stage lung adenocarcinoma. Int J Cancer 2011;129:2445-53.

23. Dhar S, Squire JA, Hande MP, Wellinger RJ, Pandita TK. Inactivation of 14-3-3 sigma influences telomere behavior and ionizing radiation-induced chromosomal instability. Mol Cell Biol 2000;20:7764-72.

24. Chan TA, Hermeking $H$, Lengauer C, Kinzler KW, Vogelstein B. 14-3-3 sigma is required to prevent mitotic catastrophe after DNA damage. Nature 1999;401:616-20.

25. Tsvetkov $P$, Reuven N, Shaul Y. Ubiquitin-independent p53 proteasomal degradation. Cell Death Differ 2010;17:103-8.
26. Nagata Y, Anan T, Yoshida T, Mizukami T, Taya Y, Fujiwara T, et al. The stabilization mechanism of mutant-type p53 by impaired ubiquitination: the loss of wild-type p53 function and the hsp90 association. Oncogene 1999;18:6037-49.

27. Chang TC, Liu CC, Hsing EW, Liang SM, Chi YH, Sung LY, et al. 14-3-30 regulates $\beta$-catenin-mediated mouse embryonic stem cell proliferation by sequestering GSK-3 $\beta$. PLoS One 2012; 7:e40193.

28. Casalini P, lorio MV, Berno V, Bergamaschi A, Borresen Dale AL, Gasparini P, et al. Relationship between p53 and p27 expression following HER2 signaling. Breast 2007;16:597605.

29. Besson A, West MG, Schmidt A, Hall A, Roberts JM. p27Kip1 modulates cell migration through the regulation of RhoA activation. Genes Dev 2004;18:862-76. 